\title{
Zusammenfassung und Ausblick
}

\section{Zusammenfassung}

Nachdem wir uns in diesem Lehrbuch mit den unterschiedlichsten Themen beschäftigt haben, ist es nun an der Zeit, ein Fazit zu ziehen. Welche spannenden Aufgaben erwarten uns in Zukunft und wie können wir „Privacy by Design“ in der Praxis umsetzen?

In diesem Lehrbuch haben wir uns mit unterschiedlichen Themengebieten beschäftigt. Wir haben gesehen, dass es an zahlreichen Stellen datenschutzrechtliche Probleme gibt. Außerdem haben wir Techniken und Verfahren kennengelernt, die dafür sorgen, dass der Datenschutz gewahrt bleibt. Dieser von uns präsentierte Ansatz des technischen Datenschutzes entspricht weitestgehend dem „Privacy by Design“, das in der kommenden EU-Datenschutzgrundverordnung verankert ist. Als Leser sollten Sie nun sowohl durch die Einblicke in das Datenschutzrecht erkennen können, welche datenschutzrechtliche Probleme bei der Entwicklung von Systemen lauern können, als auch über das nötige technische Know-How verfügen, um diesen Problemen von Beginn an zu begegnen und Systeme datenschutzfreundlich zu gestalten. Es warten, nicht nur aus Sicht des Datenschutzes, spannende Aufgaben auf Entwickler. Die weitere Durchdringung von IT in all unsere Lebensbereiche schreitet weiter voran: vom intelligenten Zuhause über das vernetzte Auto bis hin zur personalisierten Medizin. All diese Verfahren werden nur erfolgreich sein, wenn die Sicherheit und der Datenschutz gewährleistet sind. Wir hoffen, dass wir Ihnen in diesem Lehrbuch das spannende Thema Datenschutz mit all seinen Facetten näher bringen konnten und dass Sie das Erlernte sowohl in Ihrem beruflichen, als auch im privaten Alltag anwenden können. 